\title{
Future Treatment of Heart Failure Using Human iPSC-Derived Cardiomyocytes
}

\author{
Shugo Tohyama and Keiichi Fukuda
}

\begin{abstract}
Heart transplantation can drastically improve survival in patients with a failing heart; however, the shortage of donor hearts remains a serious problem with this treatment strategy and the successful clinical application of regenerative medicine is eagerly awaited. To this end, we developed a novel method to generate human induced pluripotent stem cells (iPSCs) from circulating human $\mathrm{T}$ lymphocytes using Sendai virus containing Yamanaka factors. To establish an efficient cardiac differentiation protocol, we then screened factors expressed in the future heart site of early mouse embryos and identified several growth factors and cytokines that can induce cardiomyocyte differentiation and proliferation. Subsequent transcriptome and metabolome analysis on undifferentiated stem cells and cardiomyocytes to devise a specific metabolic environment for cardiomyocyte selection revealed completely different mechanisms of glucose and lactate metabolism. Based on these findings, we succeeded in metabolically selecting cardiomyocytes using glucose-free and lactate-supplemented medium, with up to $99 \%$ purity and no teratoma formation. Using our aggregation technique, we also showed that $>90 \%$ of the transplanted cardiomyocytes survived in the heart and showed physiological growth after transplantation. We expect that combining these techniques will achieve future heart regeneration.
\end{abstract}

\section{Keywords}

Induced pluripotent stem cell • Purification • Cardiomyocyte • Transplantation • Human

\footnotetext{
S. Tohyama $\bullet$ K. Fukuda $(\triangle)$

Department of Cardiology, Keio University School of Medicine, Tokyo, Japan e-mail:kfukuda@a2.keio.jp

T. Nakanishi et al. (eds.), Etiology and Morphogenesis of Congenital Heart Disease, DOI 10.1007/978-4-431-54628-3_4
} 


\subsection{Introduction}

Heart disease remains a leading cause of death despite recent medical advances, and heart transplantation remains the ultimate treatment for severe heart failure. However, limited donor numbers remain an unsolved problem for transplantation therapy, and both patients and clinicians hold great hope for the future success of heart regenerative cell therapies as an alternative strategy [1]. Pluripotent stem cells (PSCs) including embryonic stem cells (ESCs) and induced pluripotent stem cells (iPSCs) can self-renew infinitely and are potential mass production sources for therapeutic cardiomyocytes. In particular, human iPSCs have the huge advantage of avoiding immunological rejection after cell transplantation.

Human iPSCs were first generated from dermal fibroblasts by Takanashi and Yamanaka in 2007 [2], using a retroviral transduction system. Subsequently, the methodology for generating iPSCs has dramatically improved. We previously reported that integration-free iPSCs could be easily and rapidly generated from terminally differentiated circulating $\mathrm{T}$ lymphocytes in peripheral blood using Sendai virus [3]. Our method makes it possible to generate iPSCs from any patients including children, girls, and the very elderly by blood sampling alone.

Such cumulative advances in iPSC generation techniques should accelerate the development of applications for iPSCs generated from patients. However, many hurdles remain in realizing such applications in human heart regeneration, due largely to the assumption that one patient will require at least $1 \times 10^{9}$ cardiomyocytes to recover cardiac function (Fig. 4.1). The important steps to overcome are as follows: (1) cardiac differentiation efficiencies should be stably improved regardless of the source cell lines; (2) large-scale cultivation systems will be required to obtain the billions of differentiated cells required; (3) approximately $1 \times 10^{9}$ cardiomyocytes should be efficiently collected and residual undifferentiated stem cells should be eliminated from large-scale, mixed, differentiated cell populations; (4) purified cardiomyocytes should be functionally and electrophysiologically characterized; (5) tissue engineering technologies might need to be used prior to transplantation because dispersed transplanted cardiomyocytes have not yet achieved high survival rates [4]; and (6) confirmation of safety and efficiency with these techniques is essential in large animal models before clinical application. In this chapter, we discuss these hurdles to realizing heart regenerative therapy in more detail.

\subsection{Cardiac Differentiation from Human iPSCs}

Many approaches using ESCs have been investigated to induce cardiac differentiation. In general, the differentiation of ESCs into any cell lineage is based on the mechanism of normal early development [5]. The visceral endoderm is known to play a key role in the differentiation of cardiac precursors that are present in the adjacent mesoderm during development, and Mummery et al. [6] previously reported that human ESCs effectively differentiate into cardiomyocytes when 


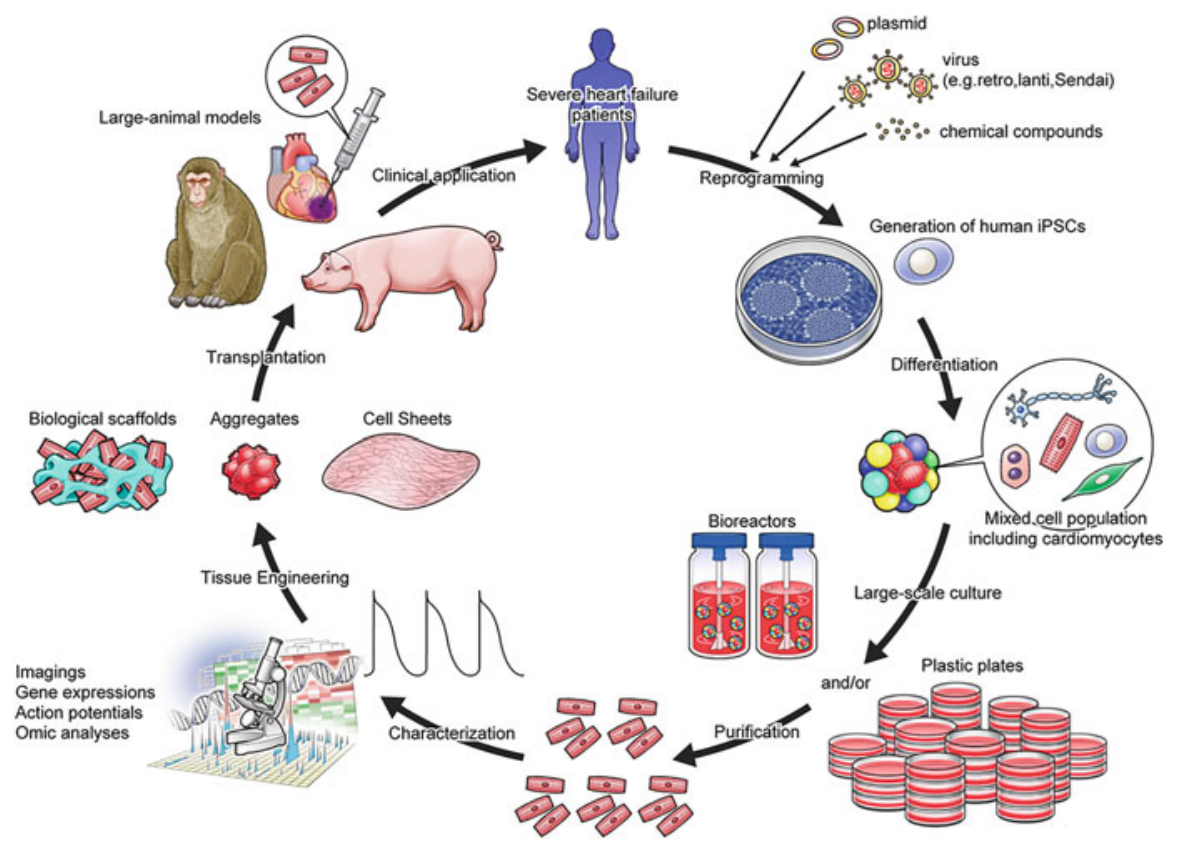

Fig. 4.1 Overview of steps to overcome for realization of heart regenerative therapy. The critical hurdles to overcome are as follows: (1) improvement of cardiac differentiation efficiencies, (2) establishment of large-scale cultivation systems, (3) purification of large-scale cardiomyocytes, (4) electrophysiological characterization, (5) utilization of appropriate tissue engineering technologies, and (6) confirmation of safety and efficiency in large animal models

cocultured with mouse visceral endoderm-like (END-2) cells. In an attempt to improve cardiac differentiation efficiencies inexpensively and easily, Takahashi et al. [6] screened a chemical compound library approved by the United States Food and Drug Administration (FDA) and found that ascorbic acid efficiently induced cardiac differentiation. However, this chemical alone might be not enough to induce cardiac differentiation from iPSCs.

Several studies have shown that various combinations of heart developmentrelated proteins including BMP, activin, Wnt, BMP inhibitor, and Wnt inhibitor induce cardiomyocytes from ESCs [7-10]. We reported that the context-dependent differential action of BMPs in cardiomyocyte induction is explained by the local action of Noggin and other BMP inhibitors and, accordingly, developed a protocol to induce cardiac differentiation of mouse ESCs through transient administration of Noggin [9]. However, to obtain hundreds of millions of cardiomyocytes, it is necessary to establish a cardiac differentiation method that is both efficient and cost-effective due to the many expensive recombinant protein factors used. To address this problem, Minami et al. [11] screened small-molecule compounds to identify those that significantly increase cardiac differentiation induction, and they revealed some inhibitors of canonical Wnt signaling as candidates. In addition, 
recently novel efficient protocols using small molecules and/or chemically defined media have been reported (Lian et al. Nat Protocols 2013;8:162-175, Burridge et al. Nat Methods. 2014;11:855-60).

Several studies then showed that induction techniques in ESCs could also be applied to iPSCs, although differentiation efficiencies were suggested to be inferior compared to ESCs. However, as such differentiation efficiencies vary greatly with different cell lines [12], further investigation is needed in the future. Furthermore, to efficiently obtain large quantities of cardiomyocytes inexpensively, it is necessary to continue refining efficient cardiac differentiation systems combined with the use of small-molecule compounds. However, despite improved cardiac differentiation efficiencies, it is inevitable that human PSC derivatives will contain not only cardiomyocytes but also undifferentiated stem cells and/or noncardiac cells because all PSCs cannot differentiate into cardiomyocytes. Therefore, to confirm safety after transplantation, it is necessary to remove noncardiac cells and undifferentiated stem cells that could cause tumors.

\subsection{Nongenetic Methods for Purifying Cardiomyocytes}

One of the biggest risks with in vitro-generated cardiomyocytes for clinical use is teratoma formation due to residual PSC contamination [13]. Current procedures for eliminating such contamination and boosting cardiomyocyte enrichment involve genetic modification $[14,15]$ and nongenetic methods using a mitochondrial dye [16] or antibodies to specific cell-surface markers [17]. However, none of these methods are ideal for the therapeutic application of PSC-derived cardiomyocytes due to insufficient stability, genotoxicity, and the use of fluorescence-activated cell sorting (FACS). To address this issue, we sought to purify cardiomyocytes efficiently and inexpensively, based on differences among cell-specific nutrition sources.

To create a metabolic environment where "residual undifferentiated stem cells cannot survive and only cardiomyocytes can survive," we performed metabolome and transcriptome analysis in neonatal cardiomyocytes and PSCs. We found that these PSCs mainly depended on activated glycolysis and actively discharged lactate into the extracellular media. In addition, biomass needed for proliferation such as amino acids and nucleic acids were actively synthesized in PSCs compared to cardiomyocytes. On the other hand, cardiomyocytes mainly depended on oxidative phosphorylation in mitochondria to obtain adenosine triphosphate (ATP) efficiently. We also performed metabolome analysis to demonstrate that other noncardiac proliferating cells also depended on glycolysis, like PSCs. Thus, cardiomyocytes and proliferating noncardiac cells including PSCs showed differences in metabolism (Fig. 4.2) that we then successfully exploited to select cardiomyocytes from human PSCs efficiently and inexpensively simply by changing the cell-specific medium to one that is glucose depleted and lactate supplemented [18]. The cardiomyocytes selected by metabolism showed normal electrophysiological properties and did not form teratoma after transplantation. 


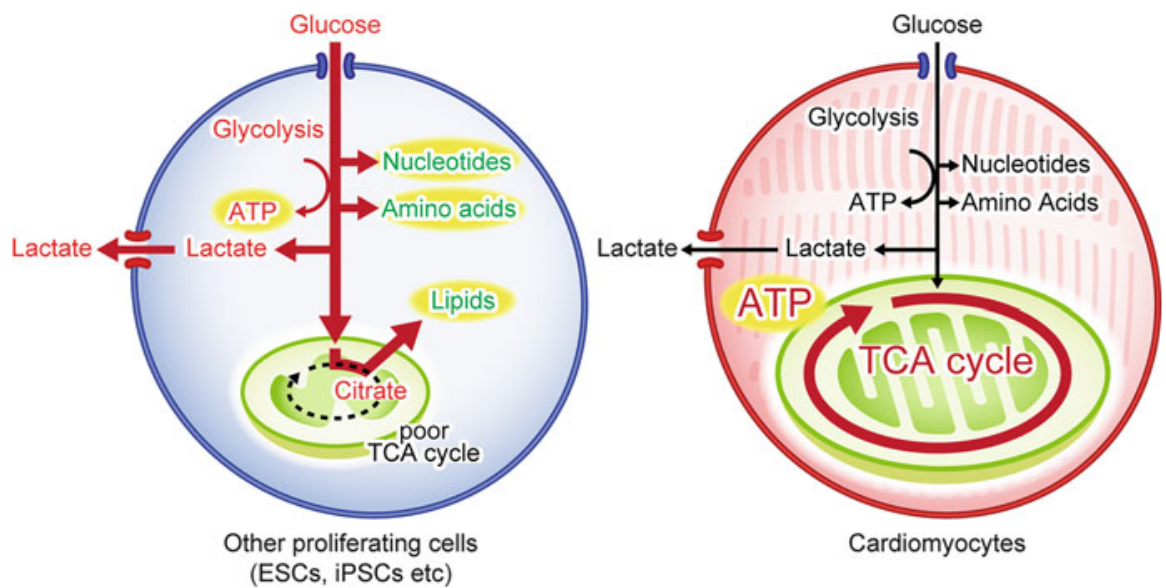

Fig. 4.2 Distinct metabolic differences between cardiomyocytes and other proliferating cells including undifferentiated stem cells. Cardiomyocytes efficiently obtain ATP mainly via oxidative phosphorylation, while other proliferating cells including PSCs obtain ATP, nucleotide, and amino acids via activated glycolysis

Furthermore, we succeeded to establish a practical culture system for generating substantial numbers of purified cardiomyocytes by combining a massive suspension culture system with a metabolic selection medium (Hemmi et al. Stem Cells Transl Med. 2014;3:1473-83).

\subsection{Transplantation of Human PSC-Derived Cardiomyocytes}

Many studies have been conducted regarding cell transplantation therapies in animal models using human PSC-derived cardiomyocytes. The survival of dispersed transplanted cardiomyocytes is reportedly very low [4], and some ingenuity is required in the method of transplantation. Our group previously reported that transplanted cell survival was dramatically improved by transplantation after formation of cardiomyocyte aggregates [16], while Laflamme et al. [9] showed a similar effect using a prosurvival cocktail that inhibited apoptosis.

With regard to the effectiveness of cell transplantation, Laflamme et al. [9] also demonstrated that transplanted human ESC-derived cardiomyocytes could improve cardiac function after 4 weeks in a rat myocardial infarction model. In addition, Shiba et al. [20] demonstrated that transplanted human ESC-derived cardiomyocytes electrically coupled to the host cardiomyocytes and suppressed arrhythmias in a guinea pig myocardial infarction model. Furthermore, with respect to cell transplantation in large animals, Kawamura et al. [19] recently reported that cardiac cell sheets comprising purified human iPSC-derived cardiomyocytes generated using our method [18] improved cardiac function in a pig myocardial infarction model. Thus, efficacies have been achieved in cell transplantation 
therapies using human iPSC-derived cardiomyocytes, although many such studies showed only short-term effectiveness. Thus, careful evaluation of the efficacy and safety of human iPSC-derived cardiomyocytes in cell transplantation over the longer term must be ongoing.

\subsection{Future Directions}

The discovery and refinement of human iPSCs generation is expected to advance not only regenerative medicine but also drug discovery and analyses of genetic disorders using patient-specific iPSCs [19]. The major and common problem remaining in this quest is securing sufficient numbers of mature and functional cardiomyocytes with high purity. To solve this problem, it is essential to develop a stable and efficient mass culture system and to establish a simple system to analyze the electrophysiological function of the generated cardiomyocytes (Fig. 4.1). Furthermore, the future realization of clinical applications using human iPSCs will necessitate a better understanding of the cell biology and techniques involved in tissue engineering generally. Only then will we be able to achieve long-term safety and efficacy in the heart failure models of large animals and finally realize human heart regenerative therapies.

Open Access This chapter is distributed under the terms of the Creative Commons AttributionNoncommercial 2.5 License (http://creativecommons.org/licenses/by-nc/2.5/) which permits any noncommercial use, distribution, and reproduction in any medium, provided the original author(s) and source are credited.

The images or other third party material in this chapter are included in the work's Creative Commons license, unless indicated otherwise in the credit line; if such material is not included in the work's Creative Commons license and the respective action is not permitted by statutory regulation, users will need to obtain permission from the license holder to duplicate, adapt or reproduce the material.

\section{References}

1. Passier R, van Laake LW, Mummery CL. Stem-cell-based therapy and lessons from the heart. Nature. 2008;453(7193):322-9.

2. Takahashi $\mathrm{K}$, et al. Induction of pluripotent stem cells from adult human fibroblasts by defined factors. Cell. 2007;131(5):861-72.

3. Seki $\mathrm{T}$, et al. Generation of induced pluripotent stem cells from human terminally differentiated circulating T cells. Cell Stem Cell. 2010;7(1):11-4.

4. Kolossov E, et al. Engraftment of engineered ES cell-derived cardiomyocytes but not BM cells restores contractile function to the infarcted myocardium. J Exp Med. 2006;203(10):2315-27.

5. Srivastava D. Making or breaking the heart: from lineage determination to morphogenesis. Cell. 2006;126(6):1037-48.

6. Takahashi $\mathrm{T}$, et al. Ascorbic acid enhances differentiation of embryonic stem cells into cardiac myocytes. Circulation. 2003;107(14):1912-6.

7. Naito AT, et al. Developmental stage-specific biphasic roles of Wnt/beta-catenin signaling in cardiomyogenesis and hematopoiesis. Proc Natl Acad Sci U S A. 2006;103(52):19812-7. 
8. Laflamme MA, et al. Cardiomyocytes derived from human embryonic stem cells in pro-survival factors enhance function of infarcted rat hearts. Nat Biotechnol. 2007;25 (9): 1015-24.

9. Yuasa S, et al. Transient inhibition of BMP signaling by Noggin induces cardiomyocyte differentiation of mouse embryonic stem cells. Nat Biotechnol. 2005;23(5):607-11.

10. Kattman SJ, et al. Stage-specific optimization of activin/nodal and BMP signaling promotes cardiac differentiation of mouse and human pluripotent stem cell lines. Cell Stem Cell. 2011;8 (2):228-40.

11. Minami I, et al. A small molecule that promotes cardiac differentiation of human pluripotent stem cells under defined, cytokine- and xeno-free conditions. Cell Rep. 2012;2(5):1448-60.

12. Osafune $\mathrm{K}$, et al. Marked differences in differentiation propensity among human embryonic stem cell lines. Nat Biotechnol. 2008;26(3):313-5.

13. Miura K, et al. Variation in the safety of induced pluripotent stem cell lines. Nat Biotechnol. 2009;27(8):743-5.

14. Hidaka K, et al. Chamber-specific differentiation of Nkx2.5-positive cardiac precursor cells from murine embryonic stem cells. FASEB J. 2003;17(6):740-2.

15. Elliott DA, et al. NKX2-5(eGFP/w) hESCs for isolation of human cardiac progenitors and cardiomyocytes. Nat Methods. 2011;8(12):1037-40.

16. Hattori F, et al. Nongenetic method for purifying stem cell-derived cardiomyocytes. Nat Methods. 2010;7(1):61-6.

17. Dubois NC, et al. SIRPA is a specific cell-surface marker for isolating cardiomyocytes derived from human pluripotent stem cells. Nat Biotech. 2011;29(11):1011-8.

18. Tohyama S, et al. Distinct metabolic flow enables large-scale purification of mouse and human pluripotent stem cell-derived cardiomyocytes. Cell Stem Cell. 2013;12(1):127-37.

19. Egashira T, et al. Disease characterization using LQTS-specific induced pluripotent stem cells. Cardiovasc Res. 2012;95(4):419-29.

20. Shiba Y et al. Human ES-cell-derived cardiomyocytes electrically couple and suppress arrhythmias in injured hearts. Nature. 2012;489(7415):322-5. 